EESTI NSV TEADUSTE AKADEEMIA TOIMETISED. XIV KÖIDE FOOSIKA-MATEMAATIKA- JA. TEHNIKATEADUSTE SEERIA, 1965, NR. 1

ИЗВЕСТИЯ АКАДЕМИИ НАУК ЭСТОНСКОИ ССР. ТОМ ХIV СЕРИЯ ФИЗИКО-МАТЕМАТИЧЕСКИХ И ТЕХНИЧЕСКИХ НАУК. 1965, № 1

\title{
РЕГИСТР НА БЕЗДИОДНЫХ ПЕРЕКЛЮЧАЮЩИХ ЭЛЕМЕНТАХ ХЭЛКЕНА
}

Одной из проблем, возникающих при конструировании схем бездиодной магнитной логики, является, несомненно, проблема усиления сигнала при помощи схемы на пассивных нелинейных элементах - ферритовых магнитопроводах с прямоугольной петлей гистерезиса.

Как уже доказано автором ['], предельное усиление схем без гашения потока на сопротивлении составляет $0,7358 H_{c} / s_{w}$ неn/мк сек; при использовании же схем с гашением потока на сопротивлении эта величина уменьшается в четыре раза.

Возможность более полного использования усилительных свойств феррита в схемах без гашения потока на сопротивленин может служить веской причиной исследования именно этой разновидности схем бездиодной магнитной логики (БМЛ).

\section{Возможности снижения требований к качеству феррита и к источникам импульсного питания}

Были рассмотрены следующие схемы БМЛ, относящиеся к схемам без гашения потока на сопротивлении:

1) схема Бенниона-Крейна на многоотверстных трансфлюксорах [2];

2) схема Иохельсона на кольцевых сердечниках [3];

3) схема Инджельберта на кольцевых сердечниках [4];

4) схемы Хэлкена на трансфлюксорах и кольцевых сердечниках $\left[{ }^{5,6}\right]$.

Во всех этих схемах применяется подмагничивание магнитопроводов. Для предотвращения стирания информации напряженность подмагничивающего поля должна быть равна или ниже порогового значения напряженности поля, при котором начинаются необратимые процессы перемагничивания. Обозначим это пороговое значение через $H_{n}$.

Однако во всех этих схемах величина напряженности магнитного поля, перемагничивающего сердечники, ограничена сверху некоторым значением $H_{\text {макс }}$ В целях сравнения характеристик перечисленных схем величина $H_{\text {макс }}$ выражалась в единицах $H_{\mathrm{n}}$. Полученный показатель кратности $h$ позволил отделить характеристики феррита от характеристик изучаемой схемы и был применен для сравнения схем БМЛ по ширине допустимых пределов изменения тока подмагничивания, величин импульсов тока перемагничивания и коэрцитивной силы ферритовых сердечников.

Нижний предел изменения тока перемагничивания сердечников определяется характеристиками феррита и не зависит от характеристик схемы. Зато верхний предел зависит от характеристики $h$. Следователь-

Содержание настоящей статьи доложено на IX Всесоюзном совещании по магнитным элементам автоматики и вычислительной техники в сентябре 1964 г. в г. Каунасе. 
но, единственным способом расширения пределов допустимых изменений амплитуд импульсов питания является применение схем БМЛ с возможно более высоким значением $h$. В таблице приведены значения $h$, вычисленные для схем БМЛ. Через $n$ обозначен коэффициент размножения информации схемы.

Для сравнения напомним, что значение $h$ для ферритовой памяти (двухкоординатная выборка с совпадением двух полутоков) равно двум. Учитывая опыт разработки и эксплуатации ферритовых запоминающих устройств, схемы со значением $h<2$ можно считать неперспективными. таблица

\begin{tabular}{l}
\hline Схемы \\
Схема Иохельсона \\
Схема Крейна на элементах \\
сАД с $n$ выходными отвер- \\
стиям \\
Схема Хэлкена на кольцевых \\
сердечннках \\
Схема Инджельберта \\
Схема Хэлкена с однократным \\
рчитыванием на трансфлюксо-
\end{tabular}

Значение $h$
$<3$

3

При $n=1$

$h=4,24$

При $n=2$

$h=2,62$

Tо же

\section{Ячейки Хэлкена}

В настоящей работе не рассматриваются проблемы реализации логических операций в схемах БМЛ, тем более, что эти вопросы не отличаются от таких же, возникающих при реализации логических операций в ферродиодных и ферротранзисторных схемах, но рассматриваются проблемы конструирования схем БМЛ с высоким значением $h$ при $n=2$.

Из таблицы видно, что наиболее высоким значением $h$ обладает схема Хэлкена на кольцевых сердечниках с неоднократным считыванием сигнала. Поэтому в качестве основной схемы была изучена схема Хэлкена, изображенная на рис. $1 a$ (схема цепей, связывающих сердечники, и схема прошивки шин питающих импульсов).

Существует возможность выбрать параметры ячейки (рис. 1a) таким образом, что одновременно обеспечены и максимум параметра $h$ и максимальное быстродействие. Схема оптимизирована в упомянутом смысле тогда, когда сердечники $1,1^{\prime}, 2,3$ и 4 идентичны и имеют одинаковое количество витков.

Режим работы ячейки следующий. Сигнал «1» записывается в ячейку во время тактов $1,2,3$ и 4 , чередующимися по полярности импульсами тока на выходе. Положительный (направление: верхняя входная клемма - нижняя входная клемма) импульс тока на входе перемагничивает сердечники 2 и 3 , а отрицательный - сердечники 1 и $1^{\prime}$. В результате все сердечники, кроме сердечника 4 , перемагничены в состояние « 1 ». В случае записи нуля в ячейку сигналов на входе нет и все сердечники остаются в состоянии «0».

Считывание сигнала происходит во время тактов $5,6,7$ и 8 . Во время пятого такта сердечник 3 перемагничивается в состояние «0», на выходе появляется положительный импульс и сердечник 4 перемагничивается в состояние «1». Во время шестого такта сердечник 4 перемагничивается в состояние «0» и на выходе появляется отрицательный импульс. Седьмой такт ничем не отличается от пятого; во время восьмого такта все сердечники ячейки переключаются в состояние «0», т. е. происходит стирание записанной в ячейки информации. Следовательно, вся система двухфазная, по четыре такта в фазе. Импульсы перемагничи- 


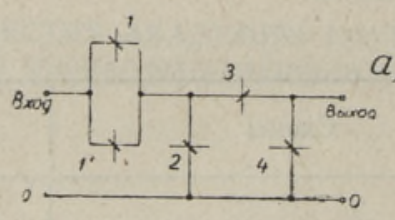

a)
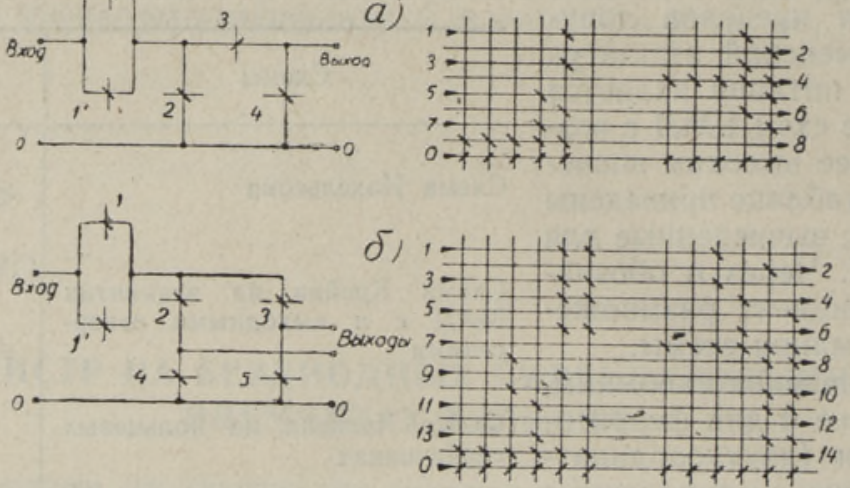

Рис. 1. Ячейка Хэлкена с одним (a) и двумя (б) выходами: слева - схема соединения обмоток; справа - схема шин тактовых импульсов и тока подмагничивания.

вания сердечников подводятся к сердечникам по шинам (см. рис. 1a). Временная диаграмма импульсов следующая: за импульсом в шине 1 следует импульс в шине 2 и т. д. После импульса в шине 8 следует импульс в шине 1 , и все повторяется. По шине 0 протекает ток подмагничивания сердечников.

Для создания запаса усиления, компенсирующего потери в цепях передачи потока, применены четыре считывающих импульса. Так как полезный поток передается только во время первого и третьего тактов, то получается двухкратный запас по усилению потока.

Весь регистр был выполнен на стандартных ферритовых сердечниках размером $2 \times 1,4 \times 0,9$ мм марки ВТ-1. Наряду с одновитковымн обмотками (в цепях шин подмагничивания и импульсов тока) на сердечниках применялись и 6-витковые обмотки (в цепях передачи потока).

Сердечники для регистра были отобраны по значению порога необратимых процессов перемагничивания и по перепаду потока при полном перемагничивании; в основном использовались сердечники с порогом $300 м a \pm 5 \%$ и с перепадом магнитного потока 15 мкс $\pm 10 \%$. Коэффициент переключения и прямоугольность петли гистерезиса не проверялись.

Регистр имел 40 последовательно включенных ячеек. В первую ячейку через вибрирующий контакт реле записывался сигнал «1». Таким образом создавались последовательности нулей и единиц, которые прошли через весь регистр.

Испытание прибора показало следующее:

1. Неоднократное считывание сигнала осуществляется весьма эффективно. Передача магнитного потока между сердечниками 3 и 4 происходит без заметных потерь. При увеличении числа тактов считывания до шести последний выходной импульс имеет еще величину порядка $70-80 \%$ по сравнению с первым (по потоку).

2. Передача магнитного потока в принимающую ячейку происходит весьма эффективно. Уже первый такт перемагничивает сердечник 3 почти полностью; второй такт стирает около 5-20\% потока, накопленного в сердечнике 3 . Третий такт домагничивает сердечник 3 почти полностью в состояние «1».

3. Усиление потока в ячейке весьма мало зависит от параметров питающих импульсов. Ширина допусков установки значений токов составляет величину порядка $\pm 20 \%$, без заметного влияния на усиление потока. 


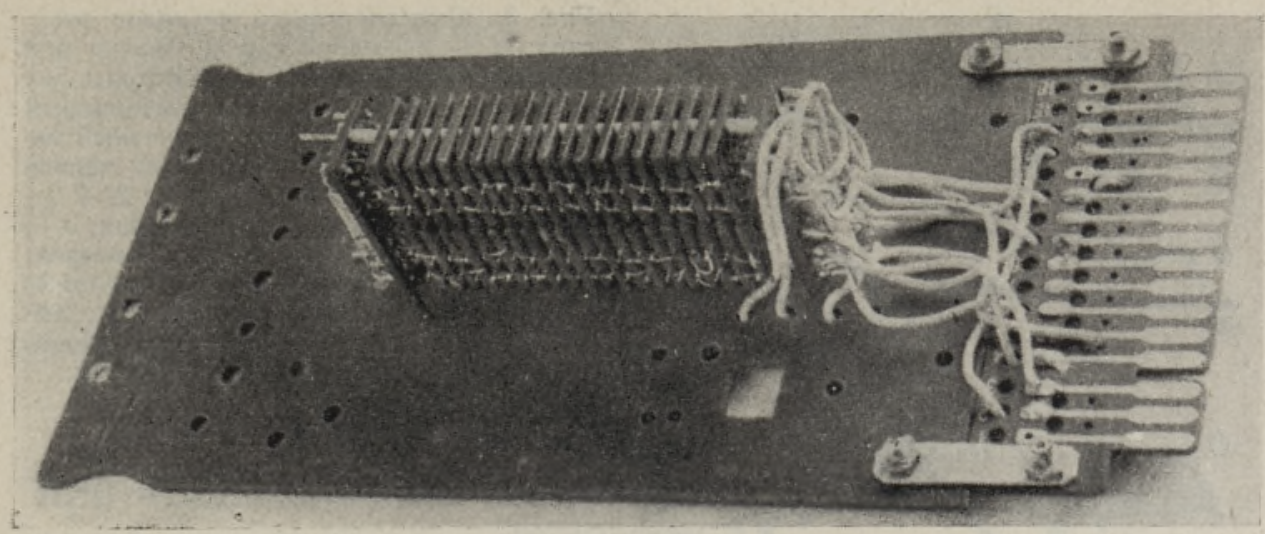

Рис. 2. Регистр из сорока ячеек Хэлкена. Размеры регистра $60 \times 34 \times 16$ мм.

4. Устойчивость системы зависит главным образом от нулевых помех. Единственный эффективный способ для подавления последних уменьшение тока подмагничивания. При этом наблюдается сильное колебание уровня нулевых помех вдоль регистра, что указывает на неодинаковые характеристики ячеек и, по-видимому, на недопустимость применения сердечников без проверки их на прямоугольность петли гистерезиса.

5. Увеличение количества считывающих импульсов до шести несколько снижает верхний предел тока подмагничивания; при этом, однако, сильно снижается и нижний предел тока подмагничивания, следовательно, расширяется область устойчивой работы регистра.

Разветвление путем подключения двух ячеек последовательно к выходу одной ячейки не может быть осуществлено, так как наблюдается неравное распределение потока между двумя принимающими ячейками, и шесть тактов считывания (коэффициент усиления потока в этом случае меньше трех) недостаточны для обеспечения прохождения сигнала без ослабления. Даже дальнейшее увеличение числа тактов считывания не дает особенного эффекта, поскольку (как уже отмечалось выше) величина сигнала с каждым тактом убывает и на седьмом-восьмом такте составляет примерно 60-70\% первоначальной величины.

Поэтому было решено использовать накопленный в сердечнике 2 поток в целях разветвления.

Как видно из схемы рис. 16, такая ячейка имеет два изолированных выхода, с которых поочередно считывают информацию (за исключением такта гашения, который действует на выходы одновременно).

Испытание такой схемы показывает, что два выхода могут действовать изолированно друг от друга; единственное взаимодействие, которое наблюдается, заключается в увеличенной помехе нуля (по сравнению с ячейкой, имеющей один выход). Поэтому схемы с разветвлением требуют сердечников с более высокой прямоугольностью петли гистерезиса.

К сожалению, регистр с разветвлением можно испытать лишь с небольшим количеством ступеней, так как из-за разветвления число ячеек в схеме при увеличении числа ступеней растет чрезвычайно быстро (в геометрической прогрессии). Поэтому не удалось привести точных сравнительных оценок обеих схем.

На рис. 2 показан внешний вид регистра из 40 ячеек; на рис. $3 a-$ осциллограммы выходного сигнала ячейки при шеститактном считывании, а также осциллограмма интегрированного сигнала сердечника 3 , 

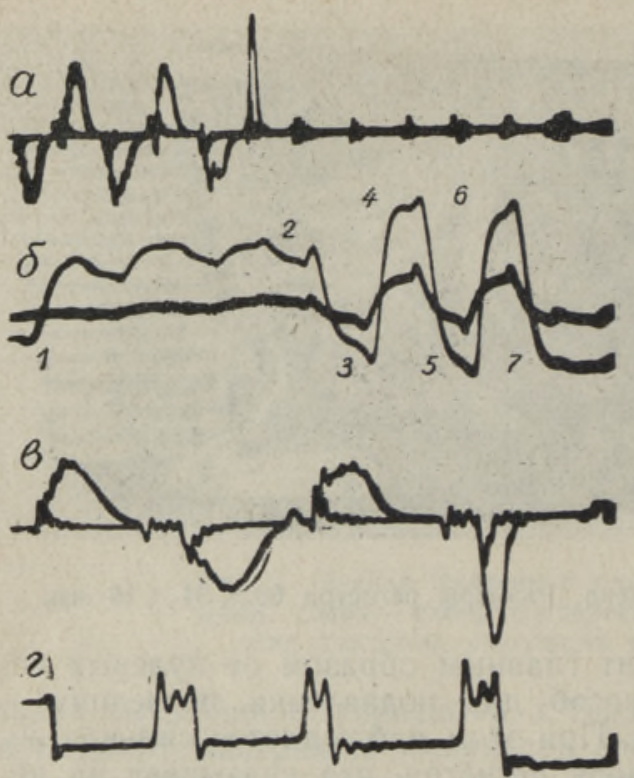

Рис. 3. Осциллограммы сигналов регистра: $a$ - выходной сигнал ячейки при шеститактном считывании, наложены сигналы «0» и «1»; 6 - интегрированный сигнал сердечника 3 (т. е. магнитный поток), участок $1-2$ изображает запись информации в ячейку, участок 2-7 процесс неоднократного считывания; в выходной сигнал ячейки при четырехтактном считывании; 2 - отрицательные импульсы тока, питающие регистр; амплитуда импульсов $2,5 a$, длительность каждого импульса 1,2-1,3 мк сек.

т. е. зависимость магнитный поток - время (рис. $3 б$ ). На рис. $3 в$ приведена осциллограмма выходног сигнала ячейки при четырехтактном считывании, а на рис. $32-$ осциллограмма импульсов, питающих регистр.

Заключение. Схема Хэлкена является работоспособной схемой бездиодной магнитной логики на кольцевых сердечниках, обеспечивающей весьма высокое быстродействие и устойчивость при достаточно широких пределах изменения питающих импульсов. Возможность применения кольцевых сердечников с диаметром 2 мм обеспечивает малогабарнтность аппаратуры и экономичность питания.

Недостатком таких схем является сложность устройств питания.

\section{Л ИТЕРАТ У Р А}

1. С ини соо М. А., Бездиодные системы магнитной логики, Сб. «Магннтные цифровые элементы», М., 1965.

2. C r a n e H. D., Proc. IRE, 47, 63 (1959).

3. Yo chels o n S. B., IRE WESCON Convention Record, P. 4, 1960, p. 82.

4. Eng e l b a rt D. C., 1959 Solid State Circuits Conference, Digest of technical papers, Philadelphia, 1959, p. 66.

5. Hölk e n U., Elektronische Rechenanlagen, 4, H. 6, 257 (1962).

6. B ill ing H., Wiss. Z. Techn. Univers. Dresden, 12, H. 1, 5 (1963).

Ннститут кибернетики

Академии наук Эстонской ССР
Поступила в редакщию 8/XII 1964

\section{SIN.ISOO}

\section{HOLLKENI DIOODIVABADEST MAGNETILISTEST LOLIVATEST ELEMENTIDEST KOOSTATUD REGISTER}

Töösageduse ja toiteimpulsside tolerantside suuruse seisukohalt käsitletakse perspektiivseid täismagnetilisi lülivaid elemente. Esitatakse ühe skeemi kirjeldus ja ühtlasi selle järgi ehitatud registri katsetulemused. Registri pikkus on 40 lülivat elementi ja ta koosneb 200 ferriitsüdamikust.

\section{SINISOO}

\section{A SHIFT REGISTER ON HÖLKEN DIODELESS SWITCHING ELEMENTS}

The paper considers various systems of diodeless magnetic logic from the standpoint of operation frequency and tolerances of pulse currents. The most favourable circuit is described. A shift register of such construction has been studied experimentally, the results being published here. The shift register had a length of 40 switching elements consisting of only two hundred $2 \mathrm{~mm}$ memory cores and connecting wire. 